\title{
The Sonographic Dimensions of the Liver at Normal Subjects Compared to Patients with Malaria
}

\author{
Moawia Gameraddin ${ }^{1,2, ~}{ }^{*}$, Amir Ali ${ }^{1}$, Mosleh Al-radaddi ${ }^{3}$, Mohaned Haleeb ${ }^{4}$, Sultan Alshoabi $^{1}$ \\ ${ }^{1}$ Faculty of Radiological Sciences and Medical Imaging, Alzaiem Alazhari University, Khartoum Bahri, Sudan \\ ${ }^{2}$ Department of Diagnostic Radiologic Technology, College of Medical Applied Sciences, Taibah University, Almadinah Almunawwarah, \\ Saudi Arabia \\ ${ }^{3}$ King Fahad Hospital, Al-Madina Almunawarah, Saudi Arabia \\ ${ }^{4}$ Madani Military Hospital, Madani City, Gezira State, Sudan
}

\section{Email addresses:}

m.bushra@yahoo.com (M. B. Gameraddin),amirmoh.ali@gmail.com (A. Ali), hanko85@hotmail.com (M. Haleeb), drraddadi@yahoo.com (M. Al-radaddi), alshoabisultan@yahoo.com (S. Alshoabi)

\section{To cite this article:}

Moawia Gameraddin, Amir Ali, Mosleh Al-radaddi, Mohaned Haleeb, Sultan Alshoabi. The Sonographic Dimensions of the Liver at Normal Subjects Compared to Patients with Malaria. International Journal of Medical Imaging. Vol. 3, No. 6, 2015, pp. 130-136.

doi: $10.11648 /$ j.jimi.20150306.14

\begin{abstract}
Determination of the liver size with ultrasound is an important process in patients with Malaria compared with the normal subjects. The purpose of this study was to determine the measurements of liver size by ultrasonography in patients with malaria compared with healthy Sudanese subjects in order to detect extend of liver enlargement. That is many tropical diseases which affect the liver size. The study population composed of 145 cases that had been selected to satisfy the study. They were 105 normal subjects (65 males 40 females) and 40 patients with Malaria from Capital Khartoum were undergone ultrasound examination of the liver. The normal subjects were divided into 5 groups according to their ages (5-102 years). The anteroposterior and craniocaudal dimensions in addition to liver span of the liver with its right and left lobes were measured using the routine ultrasonography and the mean values were recorded. Data was analyzed using statistical software program. There was highly significant difference of mean liver span of normal subjects and patients with Malaria (12.86 vs. 14.272, p-value $=0.000)$. The mean craniocaudal diameters of the right and left liver lobes were $11.932 \pm 2.02 \mathrm{~cm}$ and $9.042 \pm 2.09 \mathrm{~cm}$ for normal subjects and Malaria patients respectively. Highly significant difference existed between anterioposterior diameters of right lobes between the normal subjects and patients with Malaria (12.495 vs. 13.303, p-value $=0.000)$ There was no significant difference in anteroposterior and craniocaudal dimensions of the left lobe between the normal subjects and patients with Malaria (5.025 $\mathrm{cm}$ vs. $5.020 \mathrm{~cm}, \mathrm{p}$-value $=0.938$ and $9.079 \mathrm{~cm}$ vs. $9.042 \mathrm{~cm}, \mathrm{p}$-value $=0.903)$. Height was found to have significant correlation for the liver span followed by weight in the normal subjects. Malaria might not have impact on the size of the left lobe. The study concluded that ultrasound is an effective imaging tool and provides valuable data of volumetric analysis of the liver and its lobes in normal subjects and patients with Malaria which is of importance in the daily practice in radiology clinics since it was helpful in diagnosis of hepatomegaly.
\end{abstract}

Keywords: Ultrasonographic, Dimensions, Liver, Sudanese, Subjects

\section{Introduction}

The liver is the largest organ in the human body lies at the right hypochondriac region and it is divided in to a large right and small left lobes. The right and left morphological lobes of the liver can be further divided into number of small segments for each lobe. It performs a vital function related to metabolism, digestion, immunity and storage of nutrients. The liver is especially important for maintaining a normal blood glucose concentration. These functions made the body tissues alive. The liver size is influenced with many diseases and with the advent of liver transplantation; it has become clear that there is a great need for an exact determination of liver size. Calculated volume measurements made at ultrasound or at computed tomography provide the best estimates $[1,2,3]$.

Ultrasonography of the liver is one of the most common routine investigations which assess the size, texture and 
pathological changes. It is the first line of investigation for differential diagnosis of suspected liver pathologies. The measurement of the liver with ultrasound is important and useful to assess the size. The liver was routinely measured in the midclavicular line from the dome of the diaphragm and normally has a craniocaudal length of up to $16 \mathrm{~cm}$. A length over $16 \mathrm{~cm}$ usually indicates significant hepatomegaly though the bulk of the patient and configuration of the liver should be considered. If the liver measures $16 \mathrm{~cm}$ or more it is abnormal in 75 percent of patients. Longitudinal measurement alone is 87 percent accurate in measuring hepatic size. As the liver enlarges, it usually loses its sharp angular configuration becoming lobular in contour with a rounded inferior edge. If the liver length is $13 \mathrm{~cm}$ or less, it is considered to be normal in size in 93 percent of cases. Niederau et al studied the normal measurement of the liver with ultrasound; he reported that there was weak correlation between liver dimensions (longitudinal and transverse measurement) and physical data of the study population. He mentioned that longitudinal diameter of the liver alone will give too high or too low a value, respectively $[4,5,6]$.

Scanning of the viscera are carried out to know the normal dimensions and the echo patterns and deviations from normal have led to diagnosis or prediction of pathological conditions [7]. Liver size varies widely according to age. Many diseases can affect its size, ranging from infective processes to malignant disorders. Palpation and percussion are the standard bedside techniques to document liver size, but are far from accurate to detect small increase in size $[8,9]$. However, available data are limited for the liver, which causes difficulty in defining hepatomegaly sonographically.

Determination of the normal liver dimensions plays a great role in diagnosis of hepatomegaly as there are various diseases in Sudan may cause enlarged liver. In Sudan, Malaria is regarded as one of the most common tropical disease and most of the infected people presented with hepatomegaly. . It is a major health problem and Plasmodium falciparum causes approximately 600,000 deaths each year and the vast majority of the burden of malaria mortality is in sub-Saharan African countries [10]. Diagnosis of malaria remains a major problem in endemic regions especially in the absence of a gold standard s [11, 12]. Several studies have examined the role of ultrasound in determining hepatomegaly which is a common complication of Malaria. In our study we have compared the normal sonographic measurement of the liver in normal subjects with the patients infected with Malaria. Sowunmi studied hepatomegaly in acute Malaria and reported that hepatomegaly was more common than splenomegaly and there was positive correlation between liver enlargement and malaria [13].

There are few anthropometric studies on the normal measurements of liver by ultrasonography in Sudan and therefore, it was thought pertinent to undertake the present study, to assess the normal measurements of liver in the Sudanese population in comparison with patients infected with Malaria. In literature, most of the studies use the liver spam to assess the liver size without ignorance the other measurements of liver lobes. In this study, we aim to provide new parameters of measurement of liver; anteroposterior and craniocuadal diameters of the right lobe are similar to liver span to evaluate normal and abnormal subjects.

The sonographic measurement of the liver is very accurate and there were several studies tested the reliability of ultrasound in assessing hepatic diseases such as hepatocellular carcinoma, hepatic fibrosis, hepatic metastases and dysplastic nodules which might affect the size of the liver. Elstein et al [14] have studied the accuracy of ultrasound in assessing spleen and liver size in patients with Gaucher disease compared to computed tomographic measurements; he concluded that the two measurement showed a high degree of correlation within a broad range for both spleen and liver volumes. This result confirmed that reproducibility of ultrasound is seemed to be similar to computerized tomography. Another important study concerning reproducibility of sonographic assessment of liver fetal length in diabetic pregnancies reported that the intraobserver SD was $3.06(95 \% \mathrm{CI}, 2.68-3.59) \mathrm{mm}$; the interobserver SD was $2.17(95 \% \mathrm{CI}, 0.59-4.83) \mathrm{mm}$; the intraobserver correlation was $0.77(95 \% \mathrm{CI}, 0.63-0.87)$, and the interobserver correlation was 0.84 (95\% CI, 0.51-0.99) [15].

\section{Materials and Methods}

\subsection{Study Design and Population: This Is a Descriptive Cross-Sectional Study Which Was Conducted in Khartoum State}

There were 145 cases had been categorized into two groups; 105 normal healthy subjects, and 40 cases infected with falciparum parasite of Malaria and all diseases that cause hepatomegaly had been excluded. The variables of study were the sonographic measurement of the liver with its right and left lobes. The demographic data such as age, gender, weight, height and residence had been collected using designed questionnaire.

The sonographic measurements of the liver were collected from real ultrasound images. The demographic data were collected with a designed clinical sheet. The exclusion criteria included history of hepatic or biliary diseases, alcohol consumption, diabetes mellitus, upper abdominal surgery, hepatitis $\mathrm{B} \& \mathrm{C}$, congestive heart failure, fatty or focal hepatic abnormality and all subjects with abnormal liver patterns. Patients with Malaria had been confirmed that there were no history of biliary or hepatic diseases or diabetes mellitus and all diseases that cause hepatomegaly.

\subsection{The Ultrasonographic Procedure}

The ultrasound real images had been taken with $3.5-5 \mathrm{MHz}$ using Shimadzu machine and General Electric- Logio machine. The examination was performed by three expert Sonologists. The liver with the right and left lobes had been scanned and shown in sagittal and transverse planes and then measurements were performed to get the anteroposterior (AP) diameter and 
craniocuadal (CC) diameter in addition to the liver span.

Longitudinal views had been performed including sections through mid-line when the probe is placed directly inferior to the xiphoid process and showed the lateral left lobe, aorta and inferior vena cava (IVC). The craniocaudal length of the left lobe is measured from the medial end to the falciform ligament; the AP diameter was measured from the upper margin to the lower edge (wide distance). Liver span is the widest distance measured at full inspiration, it represent the longest diameter. It was imaged and measured from the medial angle of left lobe to the lowermost inferolateral point of the right lobe as shown in figure 1. In literature, liver span describes the distance between the lower border of the liver in the mid-clavicular line obtained by palpation, and the upper border of the liver in the mid-clavicular line detected by percussion [16].

The transverse scanning was performed with the probe placed transversely in the subxiphoid area and then the probe was swept to the subcostal region with cephalic and caudal angulation. The right lobe is scanned with visualization of the portal vein including the bifurcation and gall-bladder. The widest AP diameter of the right hepatic lobe is measured as shown in figure 2 . The scanning was performed with full inspiration.

\subsection{Statistical Analysis}

The Data had been analyzed by software programmer (Statistic Package for Social Science (SPSS 16.0) using variable statistical tests. The statistical evaluation of data was mainly descriptive. The quantitative variables such as age, AP diameters of right and left lobes, craniocuadal diameters and liver spam, we used mean $\pm \mathrm{SD}$, maximum and minimum. For comparison between quantitative variables and to find relationship, Pearson correlation and Independent t-test were applied respectively. Statistical significance was assigned at $\mathrm{p}<0.05$.

\section{Results}

Sonographic measurements of the liver of the normal subjects and patients with Malaria had been performed. Table 1 to table 4 showed the data of the normal subjects while table 5 , table 6 and table7 presented the data of the patients with Malaria. Table 1 showed the general measurements of liver lobes among the study population of the normal subjects. The craniocaudal lengths of the right and left lobes were $11.932 \mathrm{~cm}$, $9.079 \mathrm{~cm}$, while the AP diameters were $12.495 \mathrm{~cm}$ and $5.025 \mathrm{~cm}$ respectively. The mean liver span was $12.86 \mathrm{~cm}$ for the normal subjects. The AP diameters measured from the age groups of 5-10, 10-20, 20-40, 40-60 and 60-102cm; the measurements were $11.05,12.49,12.95,14.03,11.76 \mathrm{~cm}$ respectively. These measurements confirmed that AP diameters increase as age advanced until the age of 60 to 102 which revealed unexpected diminished in size $(11.76 \mathrm{~cm})$ due to age effect. The craniocaudal lengths followed the same way as the AP diameters, decreased with age as shown in table 2 .

There was no significant difference of AP diameters (table 3) of right and left lobes between male and female subjects
( $p$-values $=0.389$ and 0.805$)$ respectively.

Table 4 showed the comparison of the liver span measurement with the height and weight of the study population of the normal subjects. It was observed that the height could change the liver span measurement ( $\mathrm{p}$-value $=0.000)$ and there was also positive correlation $(r=0.563)$, while the weight showed weak correlation $(r=0.439)$ but there was significant difference $(\mathrm{p}$-value $=0.000$ ).

In patients with Malaria, the mean AP diameter of right and left lobes of the liver were $13.303 \mathrm{~cm}$ and $5.020 \mathrm{~cm}$ while the craniocaudal lengths were 12.405 and $9.042 \mathrm{~cm}$ respectively. The measurement of liver spam was categorized in groups as shown in table 6. Most of liver span measurement was more than $15.5 \mathrm{~cm}$ which is regarded to be enlarged.

The AP diameters of right lobe of the liver at Malaria patients was significantly higher than AP diameter of the normal subjects $(13.303$ vs. $12.495 \mathrm{~cm}$, p-value $=0.000)$, while the craniocaudal lengths of malaria patients is also significantly higher than those of the normal subjects $(12.405$ vs. $11.932 \mathrm{~cm}$, p-value $=0.014$ ).

The AP diameters of the left lobe in patients with Malaria was $5.020 \mathrm{~cm}$ while in normal subjects was $5.020 \mathrm{~cm}$ which were not significantly different $(\mathrm{p}$-value $=0.938)$, while the craniocaudal lengths were 9.079 and $9.042 \mathrm{~cm}$ of the abnormal and normal subjects respectively ( $\mathrm{p}$-value $=0.903$ ) as shown in table7.

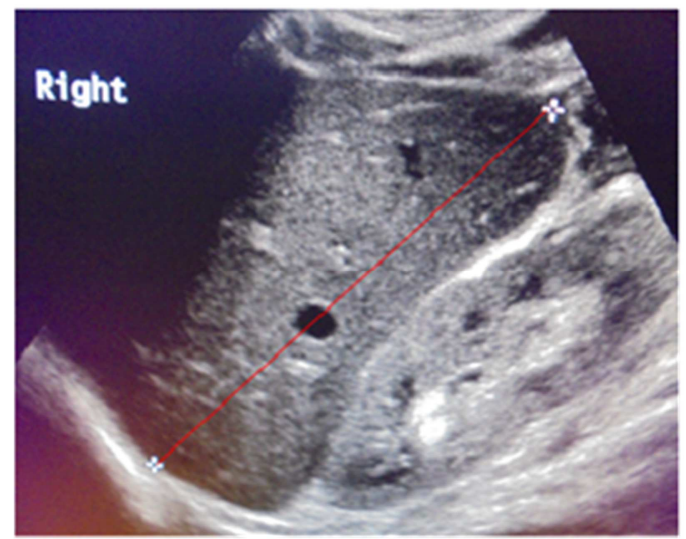

Figure 1. Sonogram illustrates liver span measurement which was performed at right mid-clavicular line of a normal subject.

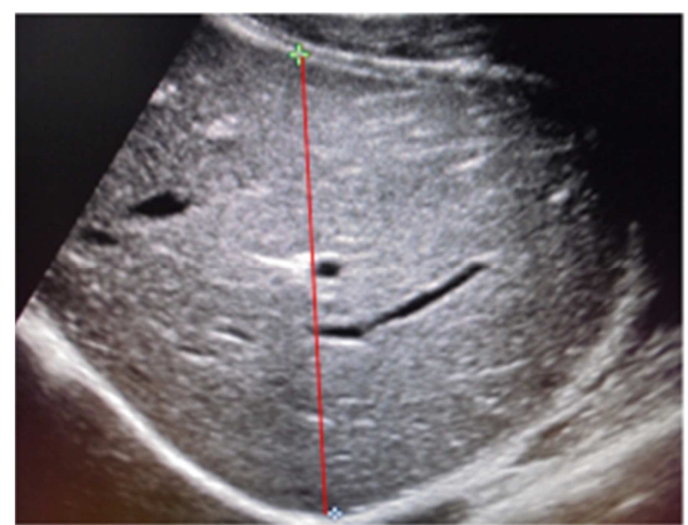

Figure 2. Sonogram showing measurement of AP diameter of enlarged right lobe of the liver of patient with malaria. 
Figure1 sonogram shows measurement of liver span, image taken with Toshiba ultrasound equipment at Medani Hospital. Figure2 sonogram shows enlarged right lobe of patient with malaria, image taken with Sonyance4 ultrasound equipment at Medani Hospital.

Table 1. The measurements of the liver lobes among the study population of the normal subjects.

\begin{tabular}{lllllll}
\hline variables & Age (years) & $\begin{array}{l}\text { CC diameter of } \\
\text { the Rt. lobe }\end{array}$ & $\begin{array}{l}\text { CC diameter of } \\
\text { the Lt. lobe }\end{array}$ & $\begin{array}{l}\text { AP diameter of } \\
\text { the Rt. lobe }\end{array}$ & $\begin{array}{l}\text { AP diameter of } \\
\text { the Lt. lobe }\end{array}$ & $\begin{array}{l}\text { Height (cm) } \\
\text { Weight (Kg) }\end{array}$ \\
\hline Total number & 105 & 105 & 105 & 105 & 105 & 105 \\
Mean & 33.733 & 11.932 & 9.079 & 12.495 & 5.025 \\
SD & 1.945 & 2.020 & 2.093 & 2.738 & 1.450 & 164.17 \\
Minimum & 5 & 3.4 & 2.3 & 1.9 & 1.5 & 18.202 \\
Maximum & 102 & 16.9 & 12.6 & 17.2 & 90 & 9.7 \\
\hline
\end{tabular}

AP: Antero-posterior

CC: Craniocaudal

SD: Standard deviation

Table 2. Measurement (cm) of the liver lobes among the age groups of study population of the normal subjects.

\begin{tabular}{|c|c|c|c|c|c|c|c|c|c|c|}
\hline \multirow{2}{*}{$\begin{array}{l}\text { Age groups } \\
\text { (years) }\end{array}$} & \multirow[t]{2}{*}{ frequency } & \multicolumn{2}{|c|}{$\begin{array}{l}\text { Mean diameter of } \\
\text { right lobe }\end{array}$} & \multicolumn{2}{|c|}{$\begin{array}{l}\text { Mean AP Diameter } \\
\text { of left lobe }\end{array}$} & \multicolumn{2}{|c|}{$\begin{array}{l}\text { Mean CC Diameter of } \\
\text { right lobe }\end{array}$} & \multicolumn{2}{|c|}{$\begin{array}{l}\text { Mean CC Diameter } \\
\text { of left lobe }\end{array}$} & \multirow[t]{2}{*}{ Mean liver spam } \\
\hline & & $\mathbf{A P}$ & SD & $\mathbf{A P}$ & SD & $\mathrm{CC}$ & SD & $\mathrm{CC}$ & SD & \\
\hline $5-10$ & 6 & 11.05 & 2.60 & 5.01 & 1.44 & 11.40 & 1.31 & 7.10 & 2.13 & \multirow{5}{*}{12.86} \\
\hline $10-20$ & 11 & 12.49 & 2.70 & 5.03 & 1.45 & 10.72 & 1.50 & 7.11 & 2.14 & \\
\hline $20-40$ & 57 & 12.95 & 2.22 & 5.13 & 1.24 & 12.20 & 1.77 & 7.17 & 1.87 & \\
\hline $40-60$ & 22 & 14.03 & 2.10 & 5.77 & 1.61 & 12.80 & 1.97 & 8.08 & 2.06 & \\
\hline $60-102$ & 9 & 11.76 & 2.38 & 4.50 & 1.19 & 10.67 & 1.38 & 8.16 & 1.34 & \\
\hline
\end{tabular}

AP: Anteroposterior

CC: Craniocaudal

SD: Standard deviation

Table 3. Comparison of the right and left lobes (anteroposterior) diameters between male and female of normal subjects.

\begin{tabular}{llllll}
\hline & Gender & total & Mean & SD & P-value \\
\hline & male & 65 & 12.677 & 2.570 & \multirow{2}{*}{0.389} \\
AP right lobe & female & 40 & 12.200 & 3.003 & \\
AP of left lobe & male & 65 & 5.052 & 1.524 & \multirow{2}{*}{0.805} \\
& female & 40 & 4.980 & 1.340 & \\
\hline
\end{tabular}

AP: Antero-posterior

SD: Standard deviation

Table 4. Comparison and correlation between the liver span and height \& weight of the normal subjects.

\begin{tabular}{lllllll}
\hline & parameter & mean & Total & SD & correlation & P-value \\
\hline & Height & 164.171 & 105 & 18.202 & & \\
Pair 1 & Liver spam & 13.476 & 105 & 1.969 & & 0.000 \\
Pair 2 & Weight & 64.298 & 105 & 15.327 & 0.439 & 0.000 \\
\hline
\end{tabular}

Table 5. The measurements (cm) of liver lobes in patients with Malaria.

\begin{tabular}{lllll}
\hline $\begin{array}{l}\text { Causes of } \\
\text { hydronephrosis }\end{array}$ & Minimum & Maximum & Mean & $\begin{array}{l}\text { standard } \\
\text { deviation }\end{array}$ \\
\hline $\begin{array}{l}\text { AP diameter of the } \\
\text { right lobe }\end{array}$ & 10.0 & 16.4 & 13.303 & 1.2636 \\
$\begin{array}{l}\text { AP diameter of the left } \\
\text { lobe }\end{array}$ & 2.7 & 10.0 & 5.020 & 1.5154 \\
$\begin{array}{l}\text { Craniocaudal length of } \\
\text { the right lobe }\end{array}$ & 10.0 & 14.9 & 12.405 & 1.1618 \\
$\begin{array}{l}\text { Craniocaudal length of } \\
\text { the left lobe }\end{array}$ & 5.7 & 12.7 & 9.042 & 1.8782 \\
$\begin{array}{l}\text { Liver span } \\
\text { Total number of }\end{array}$ & 11.5 & 16.7 & 14.272 & 1.4772 \\
patients & 40 & & \\
\hline
\end{tabular}

Table 6. The measurements of liver span in patients with Malaria.

\begin{tabular}{lll}
\hline $\begin{array}{l}\text { Measurements of liver } \\
\text { span }\end{array}$ & Number of cases & Percentage \% \\
\hline $11-12.5 \mathrm{~cm}$ & 7 & $17.5 \%$ \\
$12.6-13.5 \mathrm{~cm}$ & 7 & $17.5 \%$ \\
$13.6-14.5 \mathrm{~cm}$ & 9 & $22.5 \%$ \\
$14.6-15.5 \mathrm{~cm}$ & 7 & $17.5 \%$ \\
$>15.5 \mathrm{~cm}$ & 10 & $25 \%$ \\
\hline
\end{tabular}

Table 7. Comparison of measurements of liver lobes between normal subjects and patients with Malaria.

\begin{tabular}{|c|c|c|c|}
\hline Measurements & $\begin{array}{l}\text { The mean values } \\
\text { at Normal } \\
\text { subjects }\end{array}$ & $\begin{array}{l}\text { The mean values } \\
\text { at Malaria } \\
\text { patients }\end{array}$ & P-value \\
\hline $\begin{array}{l}\text { AP diameter of the } \\
\text { right lobe }\end{array}$ & 12.495 & 13.303 & 0.000 \\
\hline $\begin{array}{l}\text { AP diameter of the } \\
\text { left lobe }\end{array}$ & 5.025 & 5.020 & 0.938 \\
\hline $\mathrm{CC}$ of the right lobe & 11.932 & 12.405 & 0.014 \\
\hline $\mathrm{CC}$ of the left lobe & 9.079 & 9.042 & 0.903 \\
\hline Liver span & 12.86 & 14.272 & 0.000 \\
\hline
\end{tabular}

\section{Discussion}

Ultrasonography is one of the most common imaging methods which are used in routine practice [17].There have been quite a few previous reports giving the standard size of liver by ultrasound, but none has been done in the Sudanese population. In contrast to previous studies which were mainly done on the foreign population, our data was obtained from a large age group (5 - 102 years) of healthy subjects of the 
Sudanese population. These had been compared with the measurements of patients with Malaria to detect hepatomegaly. Liver size may give information about the diagnosis and the course of gastrointestinal diseases. Thus, the determination of normal liver size can be significant. The literature includes few methods for sonographic determination of liver size. The application of different measurement methods has resulted in various estimates of normal liver size [18]. Longitudinal (carniocaudal) hepatic diameter is the most commonly applied and predominant clinical method of estimating liver size in routine diagnostic situations $[19,20]$. So, approximately no study utilized the measurements of the right and left lobes of the liver as indicators of enlargement.

As well, it proved to be the best measured diameter in differentiating between healthy and diseased livers. Therefore, we chose to use liver span, craniocaudal and anterior posterior diameters for liver size measurement in this study. Our results provide a standard set of normal range of liver size in the normal subjects and patients with Malaria as determined by ultrasonography. In this study, we examined the efficiency sonographic measurement of the liver in normal subjects and patients with Malaria.

In our study the mean or average liver size in the normal subjects was $12.86 \mathrm{~cm}$ compared to $10.5 \mathrm{~cm}$ and $12.3 \mathrm{~cm}$ in Niederau et al.'s and Tarawneh et al.'s studies respectively, our value is higher. This difference may be attributed relatively to the large age group in our study. Patzak et al reported higher value $(15.0 \pm 1.5)$ compared to our value [21]. This indicates that liver spam above $15.1 \mathrm{~cm}$ is regarded enlarged. Consequently, in our study there were 13 patients out of 40 cases of malaria their liver span above $15.1 \mathrm{~cm}$ and considered enlarged.

Kratzer et al. conducted a similar prospective study on a larger population to establish normal value for liver diameter at mid-clavicular line and to determine the influence of sex, height, BMI and alcohol consumption on liver size. In their sample, the average measured liver diameter at mid-clavicular line was 14.0 $\mathrm{cm}$ compared to $12.86 \mathrm{~cm}$ in our study [22]. In comparison of our study, we found the average liver size at patients with malaria was $14.272 \mathrm{~cm}$ which is slightly higher than normal.

In the present study, we found that there was no statistical significant difference between males and females at measurement of AP diameters of the right and left lobes of the liver. In a previous study done by Mittal and Chowdhary [23], the average diameter of right lobe of the liver of males was found to be $12.99 \pm 0.76 \mathrm{~cm}$ and it was $12.66 \pm 1.07 \mathrm{~cm}$ for females. The average diameter in males was significantly higher than in females. In adults, the diameter was $11.84 \pm$ $1.03 \mathrm{~cm}$, while in elderly subjects the diameter was 13.06 $\pm 0.65 \mathrm{~cm}$. The diameter obtained by Mittal and Chowdhary in their studies was comparatively slightly lower in adult and higher in elder than that obtained by our study. Our data for the right lobe of liver was even similar to the study done by Niederau et al.

In the present study of normal subjects, we found that the average craniocaudal diameter of the left lobe of the liver was $9.079 \mathrm{~cm}$. This finding was approximately consistent to the study done by Niederau et al which was $8.3 \pm 1.7 \mathrm{~cm}$, but the it was nearly similar to the study done by Mittal and Chowdhary [23]. They reported the diameter of the left lobe to be $9.75 \pm 0.61 \mathrm{~cm}$, which is not higher in comparison to that obtained by our study. In patients with Malaria, the AP and craniocaudal diameters of left lobe was not significantly different from normal subjects $(9.079$ vs. $9.942 \mathrm{~cm}$, p-value= 0.903). This result reflects that the size of the left lobe is not influenced with Malaria thus did not enlarge.

In this study we compare anteroposterior diameters for right and left lobes(of normal subjects) between the male and female, we found no significant differences in liver measurements ( $p$-values were 0.389 and 0.805 respectively) in agreement with Da Silva et al.'s study [26]. This is not true in the literature. In a study of 915 healthy adults, Niederau and colleagues found a significant difference between genders, but they did not specify the measurement values [19]. In a study of 116 adults, Castell concluded that gender is a determining factor for liver size [27]. They discovered that men have larger liver size than women and they even proposed a formula for correcting liver measurement by clinical percussion for each gender. In a sample of 2,080 adults, Kratzer and colleagues found a significant difference for ultrasound liver measurement between male and female individuals [21]. Mean value for men was $14.5 \pm 1.6 \mathrm{~cm}$ and $13.5 \pm 1.7 \mathrm{~cm}$ for women. In a study of 307 children aged between 5 days and 16 years, Konus and colleagues, however, found no significant size difference in hepatic lobes [28]. In comparison of patients with malaria, we found the AP and craniocaudal diameters of the right lobe revealed high significant difference (12.495 vs. $13.303 \mathrm{~cm}, \mathrm{p}$-value $=0.000$ and 11.932 vs. $12.40 \mathrm{~cm}, \mathrm{p}$-value $=$ 0.014). This results indicates that right lobe of the liver is more sensitive to enlargement than the left lobe; AP and craniocuadal measurements could be regarded as accurate indicators to assess liver size.

The study revealed that the liver span of the patients with Malaria normal subjects is significantly higher than that of the normal subjects $(12.86$ vs. $14.272 \mathrm{~cm}, \mathrm{p}$-value $=0.000)$. This is attributed to the infection of plasmodium falciparum which causes hepatomegaly. A study conducted by Zha et al had studied ultrasound diagnosis of malaria; examination of liver, spleen and optic nerve sheath diameter. He confirmed that hepatomegaly $>15.1 \mathrm{~cm}$ is useful since it procures as a specificity of $75 \%$. He reported hepatomegaly $(>15.1 \mathrm{~cm})$ in 16 cases out of 40 patients with malaria and the average size was $14.76 \mathrm{~cm}$ [29]. This result is similar to our finding which showed that the average size of the liver of the infected patients was $14.272 \mathrm{~cm}$. In our study we find $25 \%$ of malaria cases the liver span measurement was above $15.5 \mathrm{~cm}$ which indicated hepatomegaly. This finding is also consistent with study conducted by Eun Mi et al studied the findings of Malaria infection with plasmodium vivax and he reported that the mean spleen and liver length were significantly higher in the Malaria group [30].

Children of the normal subject group were included in the present study, in age group 5-10 years, the measurement of craniocaudal diameters of the right and left lobes were 
consistent with a study conducted in Indian children by Dhingra et al in which liver length was $11.9 \pm 1.08 \mathrm{~cm}$ and $11.7 \pm 1.11 \mathrm{~cm}$ for the male and female respectively in the age group 8-10 years [24]. In this study, values were higher than that reported by Balcha and Admassi who conducted their study in below thirteen years old Ethiopian children [25].

In normal subjects, the present study also identified age as a factor influencing liver size. Thus, the published data regarding a correlation between liver size and age remain contradictory $[19,22]$. Zeeh and Platt showed that both liver weight and liver volume decrease with advancing age [31]. Chouke' $r$ et al. found a reduction in liver weight beginning after age 50 years. Studies by Urata et al. and Vauthey et al., which were conducted with the purpose of establishing a formula for determining liver volume, reported only a slight correlation between patient age and liver volumes [32, 33]. Corresponding to this data, we found the liver increases in size between 40 and 60 years and decreases after age 60 years. In children there was a reduction in size after age ten years.

In the present study we correlated liver spam of normal subjects to height and weight; we found that there was significantly high correlation with the liver size and height ( $p$ $<0.05)$ in the line of Konus et al study. Konus and his colleagues found that height was showed the best correlation with liver dimensions. They found that body surface area was showed a high correlation with liver, spleen and kidney dimensions, button a lesser degree. Niedarau et al and Udoaka found that there was a weak correlation between liver size and age, height, weight and BMI [34].

\section{Conclusion}

Ultrasound is very effective imaging tool to assess liver size in patients with malaria and normal subjects. The study has provided anthropometric parameters of the liver which will be useful in assessing this vital organ for any pathological enlargement or reduction in clinical practice. The sonographic measurement is very effective imaging modality for evaluating liver size at normal subjects and patients with Malaria. It is recommended to be used to screen the patients with malaria.

\section{Authors' Contributions}

Moawia B. conceived the concept. Amir wrote the first draft; Mohanad and Sultan performed the sonographic investigations. All authors involved in the data collection, critically reviewed the manuscript and all approved content of the final manuscript.

\section{References}

[1] Harlod E. Clinical Anatomy. Oxford: Blackwell publishing; 2006; pp.93-94. ed on 9-1-2015.

[2] Gyton AC., Hall JE. Textbook of Medical physiology Philadelphia: Elsevier Saunders; 2006; 859
[3] Douglas C. Clinical Methods: The History, Physical, and Laboratory Examinations. Evaluation of the Size, Shape, and Consistency of the Liver. Available from.

[4] Bisset RA., Khan AN. Differential Diagnosis in Abdominal Ultrasound. London: W.B Saunders; 2000; pp.34.

[5] Jane A. Bates. Abdominal ultrasound How, Why and When 2nd ed. London: Elsevier limited. 2004; pp. 155-70.

[6] Niederau C, Sonnenberg A, J E Muller et al. Sonographic measurement of the normal liver, spleen, pancreas and portal vein. Radiology. 1983; 149: 537-540.

[7] Marco P, Vincenzo M, Rosanna C, et al. Measurement of spleen volume by ultrasound scanning in patients with thromboocytosis: A prospective study. Blood J. 2002; 99 (11): 4228-4230.

[8] Zhang B, Lewis S. A study of the reliability of clinical palpation of the spleen. Clin Lab Haematol. 1989; (11): 7-10.

[9] Joshi R, Singh A, Jajoo N, et al. Accuracy and reliability of palpation and percussion for detecting hepatomegaly: A rural hospital based study. Indian J Gastroenterol. 2004; 23: 171-174.

[10] Mahgoub H, Gasim G I, Musa IR, Adam I: Severe Plasmodium Vivax Malaria among Sudanese Children at New Half Hospital, Eastern Sudan. Parasite Vectors. 2012; (5): 154.

[11] WHO. The health of the people: The African Regional Health Report 2006. Geneva, Swizerland: World Health Organization.

[12] Anstey NM, Price RN. Improving Case definitions for severe Malaria. Plos Med. 2007; 4: 91-92.

[13] Sowunmi A. Hepatomegaly in acute falciparum malaria in children. Trans R Soc Trop Med Hyg.1996; (90): 540-542.

[14] Elstein D, Hadas H, Azuri Y, et al. Accuracy of ultrasonography in assessing spleen and liver size in patients with Gaucher disease: comparison to computed tomography measurements. J ultrasound Med. 1997; (16): 209-211.

[15] Anderson NG, Notely E, Graham P, McEwing R. Reproducibility of sonographic assessment of fetal liver length Diabetic pregnancies. Ultrasound obstet Gynecol. 2008; (31): 529-531.

[16] Liver span, Wikipedia http://en.wikipedia.org/wiki/Liver_span Accessed 8 January 2015.

[17] Alp A, Enver S, Talat B. Sonographic assessment of the normal limits and percentile curves of liver, spleen, and kidney dimensions in healthy school-aged children. J Ultrasound Med.2005; (24): 1359-1364.

[18] 18. Sienz M, Ignee A, Dietrich C, et al. Reference values in abdominal ultrasound-liver and liver vessels in German. Z Gastroenterol. 2010; 48: 1141-1148.

[19] Niederau C, Sonnenberg A, Fritsch W, et al. Determination of liver size in clinical routine. Dtsch Med Wochenschr. 1983; 108:1599-1601.

[20] Rosenfield A, Laufer I, Schneider P. The significance of a palpable liver. A correlation of clinical and radioisotope studies. Am J Roentg, Radium Therapy, and Nuclear Med.1974; 122: 313-7. 
[21] Patzak M, Pronzner M, Oeztuerk S, et al. Assessment of liver size by ultrasonography. Journal of Clinical Ultrasound. 2014; 7: 42

[22] Kratzer W, Fritz V, Mason RA, et al. Roemerstein. Study group: factors affecting liver size: A sonographic survey of 2080 subjects. J. Ultrasound Med. 2003; 22: 1155-1161.

[23] Mittal R, Chowdhary D. A pilot Study of normal measurement of the liver and spleen by ultrasonogtaphy in the Rajasthani population. Journal of Clinical and Diagnostic Research. 2010; 4: $2733-2736$

[24] Dhingra B, Sharma S, Mishra D, et al. Normal Values of Liver and Spleen Size by Ultrasonography in Indian children. Indian Pediatrics. 2010; 47: 487-492.

[25] Balch H, Admassi D. Normal liver size measurement in Ethiopian children below thirteen years of age, Black lion hospital, Addis Ababa, Ethiopia. Ethio Med J. 2012; 50: 153-158.

[26] Da Silva R, Perrira R, Siqueira M, et al. Correlation between clinical evaluation of liver size versus ultrasonography evaluation according to body mass index (BMI) and biotypes. Rev Med Chile. 2010; 138: 1495-1501.

[27] Castell D, O'Brien K, Muench H, et al. Estimation of liver size by percussion in normal individuals. Ann Int Med. 1969; 70: 1183-1189.
[28] Konus Ö, Özdemir A, Akkaya A, et al. Normal liver, spleen, and kidney dimensions in neonates, infants, and children: evaluation with sonography. Am J Roentg. 1998; 171: 1693-1698.

[29] Yuanting Z, Michelle Z, Anjali H,et al. Ultrasound Diagnosis of Malaria: Examination of the spleen, liver and Optic nerve sheath diameter. World J Emerg Med. 2015; 6: 13-14.

[30] Eun M, Hyeon Je, chong $\mathrm{R}$ et al. Abdominal Copmputed tomography Findings of Malaria infection with Plasmodium vivax. Amj Trop med. 2010; 83: 1202-1205.

[31] Zeeh J, Platt D. The aging liver: structural and functional changes and their consequences for drug treatment in old age. Gerontology. 2002; 48: 121.

[32] Chouker A, Martignoni A, Dugas M, et al. Estimation of liver size for liver transplantation: the impact of age and gender. Liver Transpl. 2004; 10: 678.

[33] Urata K, Kawasaki S, Matsunami H, et al. Calculation of child and adult standard liver volume for liver transplantation. Hepatology. 1995; 21: 1317.

[34] Udoaka A, Enyi C, Agi C, et al. Sononlogical evaluation of the liver, spleen and kidneys in an adult Southern Nigerian population. Asian journal of medical and applied sciences. 2013; 5: 33-36. 